\title{
Multiscale modeling of oxidative degradation of C-SiC composite
}

\author{
Veera Sundararaghavan ${ }^{1}$ and Sangmin Lee ${ }^{2}$ \\ Department of Aerospace Engineering \\ University of Michigan \\ 1320 Beal Ave, Ann Arbor, MI 48109, USA.
}

\begin{abstract}
A multi-scale model has been developed for modeling thermal oxidation of ceramic matrix/carbon fiber composites used in high temperature environments. The competition between transport of oxygen and carbon dioxide between the exposed carbon fibers and the external environment is simulated using a mass conservation equation in the inert porous matrix. Oxidation at the scale of individual carbon fibers is modeled using level set method and an adaptive meshing strategy. The recession of carbon fiber at interfaces exposed to oxygen is simulated as a function of time using a coupled finite element methodology at various locations in a validated macroscopic model. The multi-scale model allows study of stress-oxidation coupling and associated mechanical property degradation in $\mathrm{C} / \mathrm{SiC}$ composites.
\end{abstract}

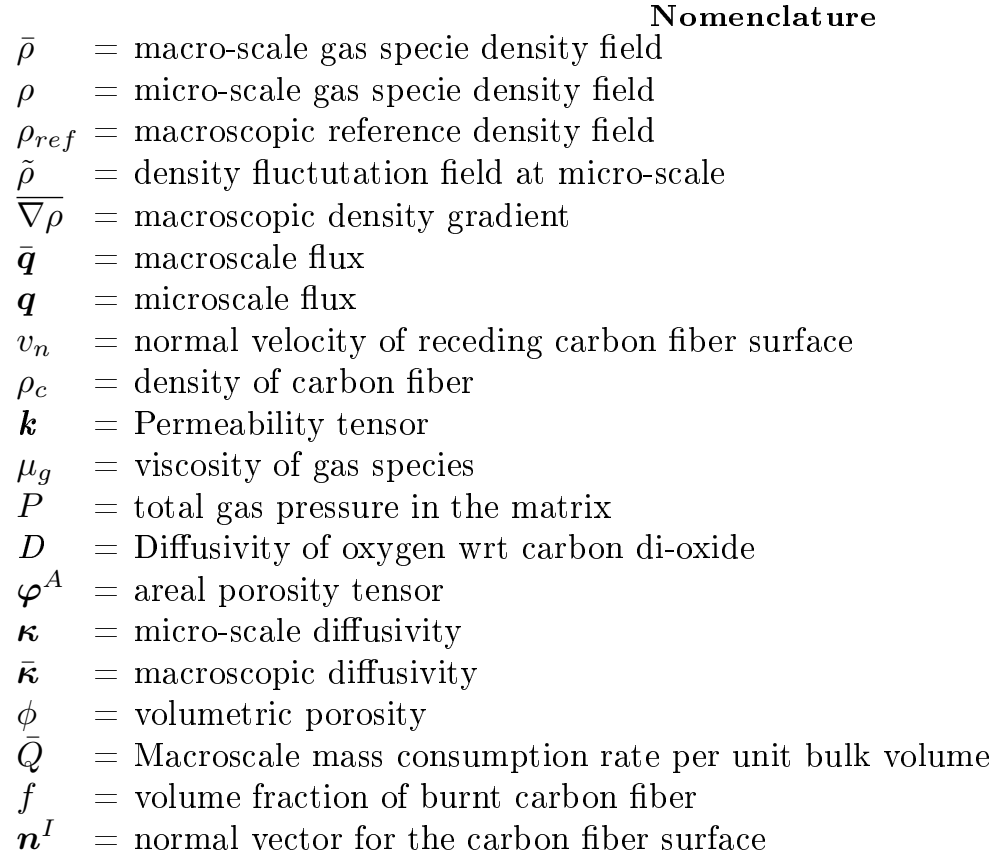

I. Introduction

Advanced launch vehicles employ ceramic matrix composites (CMCs) as structural elements in heat shields, propulsion components and other applications that encounter high temperature oxi-

\footnotetext{
1 Assistant Professor, Aerospace Engineering, Room 3025, veeras@umich.edu.

${ }^{2}$ PhD student, Mechanical Engineering, crealucu@umich.edu.
} 
dizing environments. These conditions progressively degrade the material which eventually leads to failure of the component well short of its expected life [1]. A variety of degradation mechanisms exist (Fig. 1), with the predominant one being the oxidation of $\mathrm{C}$ fibers. For moderate to high temperatures, this oxidation process is controlled by the transport of oxygen into the porous $\mathrm{SiC}$ matrix. The oxygen transport phenomena in $\mathrm{C} / \mathrm{SiC}$ composites is primarily through an interconnected void network in the matrix formed due to thermal expansion mismatch between carbon fibers and the matrix during processing. At the micro-scale, fine carbon fibers in within tows interact with oxygen and degrade at moderate to high temperatures. Initial models for studying oxidation of $\mathrm{C} / \mathrm{SiC}$ composites at micro-scales assumed a steady state diffusion through the matrix [2, 3]. While these techniques model the micro-scale, techniques for homogenizing the results for use in global analysis was not developed. In [4], a continuum level theory for modeling composite oxidation was developed. The approach models the composite as a homogeneous mixture of matrix, carbon fiber and voids. While continuum models are practical approaches for predicting the behavior of $\mathrm{C} / \mathrm{SiC}$ composites, they do not model the inherent heterogeneities involved in fiber oxidation. Multi-scale modeling by coupling macroscopic and microscopic models allow us to take advantage of both the efficiency of continuum models and the accuracy of the microscopic models.

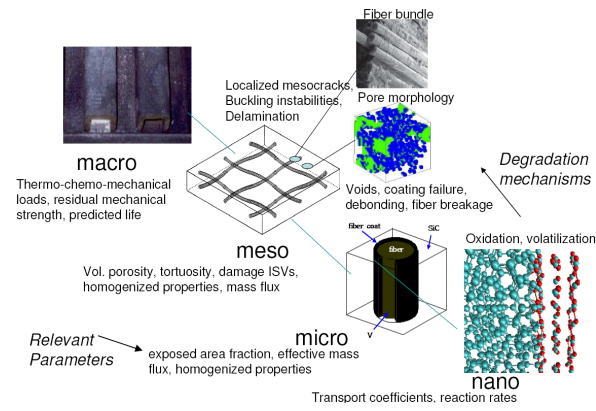

Fig. $1 \mathrm{C} / \mathrm{SiC}$ composites in extreme thermo-chemo-mechanical loading conditions are subject to a variety of degradation mechanisms. Modeling of macro-scale degradation should involve physical models at multiple length scales. In this paper, we focus on micro-scale degradation modeling using finite element analysis.

Multi-scale analysis of diffusion problems have been previously addressed using micro-scale effective properties obtained through either bounding relations[5, 6] or analytical closed-form expressions (reviewed in [7]) in a macro-scale model. These approaches were restricted to simple geometries with a simple material response, not valid when degrading interfaces are present. More recently, numerical schemes using asymptotic homogenization approaches, based on an expansion of the unknown field variable with respect to a micro-scale length parameter, have been developed to address micro-macro diffusion problems [8-10]. However, the problems considered are restricted to constant transport properties and focused on steady-state diffusion problems. Oxidation problems involve transient effects, and in addition, involve interface phenomena that have not been previously addressed in a multi-scale methodology. Computational homogenization provides an attractive avenue for computing the macroscopic response in problems with discontinuities and non-linearities. Computational homogenization is a multi-scale analysis approach in which computations are concurrently performed at two different length-scales. The macro-scale is associated with the component being modeled $\left(10^{-3}\right.$ to $\left.10^{+1} \mathrm{~m}\right)$ and the meso-scale is characterized by the underlying composite microstructure $\left(10^{-6}\right.$ to $\left.10^{-3} \mathrm{~m}\right)$. The principle of scale separation states that the characteristic length scale over which the macroscopic field variables vary, should be much larger than the size of the microscopic volume considered. In other words, macroscopic quantities are nearly constant at the level of a RVE. A representative volume element (RVE) is defined at the micro-scale and boundary conditions are defined on the RVE in terms of macroscopic quantities. Applications of such approaches for thermo-mechanical deformation have been well studied previously [11-13].

Flux discontinuities arise in several problems, the most well-studied of which is fluid solidification. In solidification problems, a flux jump (manifested as latent heat) occurs at the evolving solidification front while the overall temperature field itself is continuous in the domain. Recently, the homogenization scheme was extended for addressing such problems in our recent work [15]. Oxidation problem addressed in this work not only involves a flux discontinuity but also addition- 
ally involves a field discontinuity in the form of a jump in oxygen density field across the oxidizing interface. In this paper, we develop a fully coupled multi-scale homogenization approach for problems involving both field and flux discontinuities. The paper is organized as follows: In sections 2 and 3, a generalized scale transition for the multiscale problem is derived. The micro-scale and the macro-scale models are explained in sections 4 and 5 , respectively. In section 6 , the level set method used to model the moving interface is explained. Finally, in section 7, we discuss the methodology through an example of oxidation of a carbon composite.

\section{Computational Homogenization Approach: Micro-scale Boundary Conditions}

We consider a problem of oxidation, where carbon fibers residing in an inert matrix reacts with oxygen. The microstructure of a 2-D C/SiC composite is shown in Fig. 2. The unit cell consists of a single carbon fiber in a porous matrix occupying a volume $V$ with external boundary $S$ and outward normal $\boldsymbol{n}$. The oxidizing interface is a moving internal boundary which is initially an intact fiber-matrix interface. Material outside of the exposed carbon fiber surface occupies a volume $V^{+}$ and the exposed carbon fiber occupies a volume $V^{-}$. Here, $V^{+}$and $V^{-}$are open subsets of the total unit cell volume $V$. At the interface $S^{I}$ between $V^{+}$and $V^{-}$, the carbon fiber oxidizes further and recedes into $V^{-}$along $\boldsymbol{n}^{I}$ with speed $v_{n}$, where $\boldsymbol{n}^{I}$ is the inward normal of $S^{I}$ as indicated in Fig. 2). One such unit cell is attached to every integration point in the macro-scale finite element mesh.

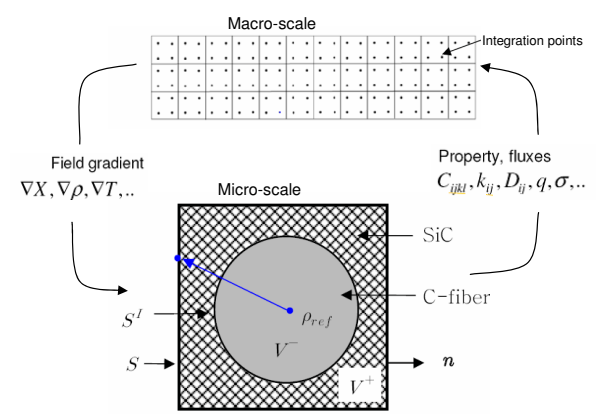

Fig. 2 Macro-scale is associated with a homogenized continuum. The macro-scale fields and field gradients are passed to the micro-scale as boundary conditions. Macro-scale fluxes and properties (at all integration points) are computed from the underlying microstructural sub-problems using averaging schemes.

To identify boundary conditions that needs to be employed at the unit-cell level, we employ the computational homogenization approach developed in [11-13, 18]. Macro-micro linking is achieved by decomposing the micro-scale field $(\rho)$ into a sum of macroscopic field and a fluctuation field $(\tilde{\rho})$ as:

$$
\rho=\rho_{\text {ref }}+\overline{\nabla \rho} \cdot \boldsymbol{x}+\tilde{\rho}
$$

Here, the coordinate $\boldsymbol{x}$ represents a point on the micro-scale relative to a reference point at the center of the unit cell. The micro-scale partial density field of species $i$ is denoted as $\rho$ and the macroscopic reference partial density field of species $i$ is denoted as $\rho_{\text {ref }}$. No subscripts (for eg. $\rho_{i}$ for species $i$ ) are used in this section to maintain generality.

In general, we denote a macroscopic counterpart of a microscopic quantity (say, $\chi$ ) as $\bar{\chi}$. In the above equation, gradient in partial density of specie $i$ at the macroscopic material point is denoted as $\overline{\nabla \rho}\left(=\nabla_{\text {macro }} \bar{\rho}\right)$. The most general assumption behind homogenization theory is that the gradient as seen at the macro-scale $(\overline{\nabla \rho})$ can be represented purely in terms of the field variables at the exterior boundary of the microstructure (Ref. [15, 16]):

$$
\overline{\nabla \rho}=\frac{1}{V} \int_{S} \rho \boldsymbol{n} d S
$$


Using the micro-scale field decomposition (Eq. 1), it can be shown that:

$$
\frac{1}{V} \int_{V} \nabla \rho d V=\bar{\nabla} \rho+\frac{1}{V} \int_{V} \nabla \tilde{\rho} d V
$$

We employ the generalized divergence theorem of the form $\int_{V} \nabla \chi d V=\int_{S} \chi \boldsymbol{n} d S+\int_{S^{I}}[|\chi|] \boldsymbol{n}^{I} d S^{I}$ in the above equation (where, $[|\chi|]$ denotes the jump in the field quantity across the evolving interface) to obtain the following relationship:

$$
\frac{1}{V} \int_{S} \rho \boldsymbol{n} d S=\overline{\nabla \rho}+\frac{1}{V} \int_{S} \tilde{\rho} \boldsymbol{n} d S+\frac{1}{V} \int_{S^{I}}([|\tilde{\rho}|]-[|\rho|]) \boldsymbol{n}^{I} d S^{I}
$$

In the above equation, $[|\rho|]$ denotes the jump in partial density of specie $i$ across the interface $\left(S^{I}\right)$ with normal $n^{I}$. The jump in field across an interface is computed as $[|\rho|]=\rho_{+}-\rho_{-}$. Here, $\rho_{+}$ and $\rho_{-}$refer to the field values in domain $V^{+}$and $V^{-}$, respectively, close to a point on the interface.

Boundary conditions at the micro-scale are identified by comparing Eq. 2 with Eq. 4. Thus, any such boundary conditions derived must satisfy the 'linking condition':

$$
\frac{1}{V} \int_{S} \tilde{\rho} \boldsymbol{n} d S+\frac{1}{V} \int_{S^{I}}([|\tilde{\rho}|]-[|\rho|]) \boldsymbol{n}^{I} d S^{I}=0
$$

A variety of boundary conditions may be derived that satisfy this constraint, however, following our previous work in [15], we focus on two possible boundary conditions (also called 'macro-micro linking assumption') as given below:

1. Taylor boundary condition involves specification of density field at all points in the microstructure. The approach is called 'Taylor model' based on similar terminology used in multi-scale deformation problems where displacements are fully specified at micro-scale. The Taylor model involves the following boundary conditions:

$$
\begin{aligned}
& \tilde{\rho}=0 \text { on } V^{+} \\
& \rho=0 \text { on } V^{-}
\end{aligned}
$$

The first term in Eq. 5 vanishes based on the first condition (Eq. 6). The second equation implies that the density of oxygen (and carbon dioxide) is negligible inside the carbon fiber. The mechanism of transport of oxygen in the carbon fiber is through lattice diffusion and this rate of diffusion is indeed negligible compared to gas phase transport (within pores) as noted in diffusion studies [17]. Comparing Eq. 7 with Eq. 1 leads to expression for $\tilde{\rho}$ inside the carbon fiber: $\tilde{\rho}=-\rho_{r e f}-\overline{\nabla \rho} \cdot \boldsymbol{x}$ on $V^{-}$. This leads to the expression for jump in the fluctuation density field at the interface:

$$
[|\tilde{\rho}|]=\rho_{\text {ref }}+\overline{\nabla \rho} \cdot \boldsymbol{x} \text { on } S^{I}
$$

Similarly, comparing Eq. 6 with Eq. 1 leads to $\rho=\rho_{\text {ref }}+\overline{\nabla \rho} \cdot \boldsymbol{x}$ on $V^{+}$. Since $\rho=0$ on $V^{-}$ (Eq. 7), we can obtain the expression for jump in field at the interface

$$
[|\rho|]=\rho_{\text {ref }}+\overline{\nabla \rho} \cdot \boldsymbol{x} \text { on } S^{I}
$$

From Eqs. 8 and Eq. 9, it can be verified that the expression $[|\tilde{\rho}|]-[|\rho|]$ in Eq. 5 also vanishes at points on the interface $S^{I}$. Thus, the Taylor assumption fully satisfies the linking condition (Eq. 5). Note that when using the Taylor linking assumption, the density fields at the micro-scale are fully specified from macro-scale quantities using the following equation:

$$
\begin{array}{r}
\rho=\rho_{\text {ref }}+\overline{\nabla \rho} \cdot \boldsymbol{x} \text { on } V^{+} \\
\rho=0 \text { on } V^{-}
\end{array}
$$


2. Homogenization boundary condition: The essential boundary condition of the following form is specified:

$$
\begin{array}{r}
\tilde{\rho}=0 \text { on } S \\
([|\tilde{\rho}|]-[|\rho|])=0 \text { on } S^{I}
\end{array}
$$

Using Eq. 11, essential boundary conditions (in the form of densities of oxygen and oxide species) are enforced at the boundaries of the unit cell as:

$$
\rho=\rho_{\text {ref }}+\overline{\nabla \rho} \cdot \boldsymbol{x} \text { on } S .
$$

The second constraint in Eq. 12 is trivially satisfied based on Eq. 1 since macroscopic fields are assumed to be continuous. In homogenization approach, the density fields are known at the external boundary and micro-scale equilibrium equations are solved using finite element analysis to completely describe the oxygen density distribution at the micro-scale.

\section{Micro-Macro linking: Transferring Fluxes and Diffusivities}

In a coupled multi-scale model, the micro-scale results are used to compute transport properties and mass flux of species at the macro-scale. This forms the basis for micro- to macro- linking. In particular, we are interested in obtaining a macroscopic flux that satisfies HillŠs macro-homogeneity condition (which relates the macroscopic flux $(\overline{\boldsymbol{q}})$ with its microstructural counterpart $(\boldsymbol{q}[15,30])$ as follows:

$$
\overline{\nabla \rho} \cdot \overline{\boldsymbol{q}}=\overline{\nabla \rho \cdot \boldsymbol{q}}
$$

The fluxes need to be derived such that the above macro-homogeneity condition is satisfied when using either Taylor or homogenization boundary conditions as follows:

- The Taylor model Using boundary conditions (Eq. 6 and Eq. 7) in the macro-homogeneity condition (Eq. 14) leads to the following:

$$
\begin{array}{r}
\overline{\nabla \rho \cdot \boldsymbol{q}}=\frac{1}{V} \int_{V} \nabla \rho \cdot \boldsymbol{q} d V=\frac{1}{V} \int_{V} \nabla\left(\rho_{r e f}+\overline{\nabla \rho} \cdot \boldsymbol{x}\right) \cdot \boldsymbol{q} d V \\
=\overline{\nabla \rho} \cdot \frac{1}{V} \int_{V} \nabla \boldsymbol{x} \cdot \boldsymbol{q} d V=\overline{\nabla \rho} \cdot \frac{1}{V} \int_{V} \boldsymbol{q} d V
\end{array}
$$

Comparing Eq. 15 with Eq. 14:

$$
\overline{\boldsymbol{q}}=\frac{1}{V} \int_{V} \boldsymbol{q} d V
$$

\section{- The Homogenization model}

We can show that a similar expression holds for the homogenization approach -Ref. [15], ie. the macroscopic flux is same as the volume averaged heat flux at the micro-scale as shown below:

$$
\overline{\boldsymbol{q}}=\frac{1}{V} \int_{S} \boldsymbol{x} q_{n} d S=\frac{1}{V} \int \boldsymbol{q} d V
$$

\section{Micro-scale model}

Since the microscopic length scale is considered to be much smaller than the scale of variation of the macroscopic temperature field, the micro-scale can be assumed to be at steady state at any instant of the macroscopic (transient) evaluation. This is a general assumption in homogenization theory due to the scale separation principle (eg. [18]). 
The microscopic mass conservation equation is given as:

$$
\nabla \cdot \boldsymbol{q}_{i}=0
$$

where, $\boldsymbol{q}_{i}$ denotes the mass flux of species $i$ and $\rho_{i}$ denotes the partial density of species $i$. The micro-scale model includes both density jump $\left(\left[\left|\rho_{i}\right|\right]\right)$ and flux jump $\left(\left[\left|\boldsymbol{q}_{i}\right|\right]\right)$ for each specie $i$ in the normal direction across the oxidizing interface. Let $\boldsymbol{v}$ represent the velocity of recession of the exposed carbon ( $\boldsymbol{v}$ is non-zero only on the evolving interface). The condition for local mass balance across the oxidizing interface (with the flux and velocity component along the normal to the interface given as $q_{n}$ and $v_{n}$, respectively) is given by the Rankine-Hugoniot condition [19, 20]:

$$
\left(\left[\left|q_{n_{i}}\right|\right]+v_{n}\left[\left|\rho_{i}\right|\right]\right)=0
$$

As described before, the primary mechanism of oxygen transport is concentration and pressure gradient driven diffusion through the pores (or cracks) in the matrix. In comparison, the species density and mass flux inside the carbon fiber is negligible (ie. $\boldsymbol{q}_{i}^{-}=\mathbf{0}$ ). Thus, the following interface flux condition can be derived to simulate mass loss of carbon fiber during oxidation:

$$
\boldsymbol{q}_{O_{2}}^{+}=-\frac{M_{O_{2}}}{M_{C}} \rho_{c} \boldsymbol{v}, \quad \boldsymbol{q}_{C O_{2}}^{+}=\frac{M_{C O_{2}}}{M_{C}} \rho_{c} \boldsymbol{v} \text { on } S^{I}
$$

where, $\rho_{c}$ is the density of the carbon fiber and $M_{i}$ denotes the molecular mass of species $i$. The sign in the above equation indicates that oxygen is consumed and carbon dioxide is released at the interface during oxidation. The presence of interface flux at the micro-scale leads to a homogenized source term (for oxygen and carbon dioxide densities) in the macroscopic model.

\section{A. Description of flow in the porous matrix at micro-scale}

The mass flux of species within the matrix with volumetric porosity $(\phi)$ is a result of both pressure gradient driven flow and concentration gradient driven flow. The net flux is represented as:

$$
\boldsymbol{q}=\boldsymbol{q}_{i}^{\alpha}+\boldsymbol{q}_{i}^{\beta}
$$

where $\boldsymbol{q}_{i}^{\alpha}$ and $\boldsymbol{q}_{i}^{\beta}$ are the mass flux of species i due to pressure gradient-driven flow and concentration gradient-driven flow, respectively.

The flux contribution within the porous matrix due to pressure gradient driven flow is given by the Darcy's equation as:

$$
\boldsymbol{q}_{i}^{\alpha}=-\rho_{i}^{p} \frac{1}{\mu_{g}} \mathbf{k} \nabla P
$$

Here, $\rho_{i}^{p}=\frac{\rho_{i}}{\phi}$ denotes the partial density of the species $i$ in the pore. In addition, $\mu_{g}$ denotes the viscosity of the gas mixture, $\mathbf{k}=k \boldsymbol{I}$ ( $\boldsymbol{I}$ is the identity matrix) denotes the second-order material permeability tensor and $P\left(=\sum_{i} P_{i}=P_{\mathrm{O}_{2}}+P_{\mathrm{CO}_{2}}\right)$ represents the total pressure of the gas mixture within the pore. The partial pressures $\left(P_{i}\right)$ for each specie $i$ are obtained using the ideal gas law ( $\rho_{i}^{P}=\frac{M_{i} P_{i}}{R T}$, where $R$ is the universal gas constant and $T$ is the temperature). Similarly, the density of the mixture within the pore can be computed as $\rho^{p}=\frac{M P}{R T}$, where $M$ is the averaged molecular weight of the gas mixture.

The concentration gradient driven mass flux in the porous matrix (based on Ref. [4]) is given using Fick's law of diffusion that involves the second-order areal porosity tensor $\left(\varphi^{A}\right)$ which is used as a measure of resistance to concentration gradient-driven flow through the pore network:

$$
\boldsymbol{q}_{i}^{\beta}=-\rho^{p} D \varphi^{A} \nabla\left(\frac{\rho_{i}^{p}}{\rho^{p}}\right)
$$

where $D$ is the diffusivity of oxygen w.r.t carbon dioxide. The areal porosity tensor can be thought of as a ratio of porosity to the tortuosity [22]. We employ the calibrated isotropic areal porosity 
from Ref. [4] for the matrix in the micro-scale calculations. The diffusivity $D$ of gas specie A w.r.t gas specie B as given by the Chapman-Enskog equation [21]:

$$
D=0.0018583 \frac{\sqrt{T^{3}\left(\frac{1}{M_{A}}+\frac{1}{M_{B}}\right)}}{P \sigma_{A B}^{2} \Omega_{D, A B}}\left[\mathrm{~cm}^{2} / \mathrm{sec}\right]
$$

where, $M_{i}$ is the molecular weight of species $i$ and $\Omega_{D, A B}$ and $\sigma_{A B}$ are computed from the LennardJones potentials of the respective species (the parameters employed are tabulated in Table 1).

Table 1 Lennard Jones potential parameters for the diffusivity between $\mathrm{O}_{2}$ and $\mathrm{CO}_{2}(k$ : Boltzmann constant)

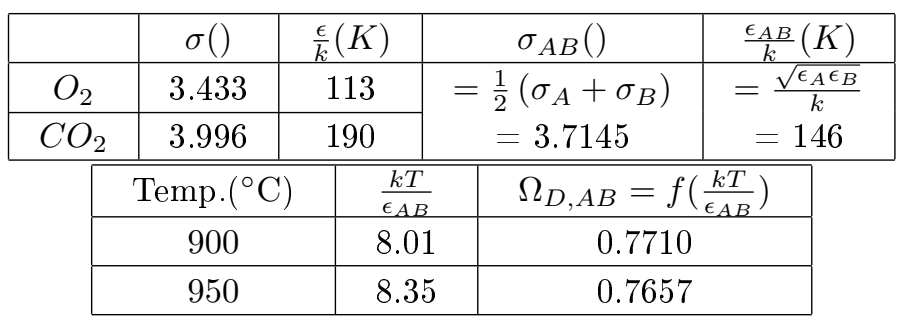

Based on Eq. 21, Eq. 22 and Eq. 23, the constitutive relationships between homogenized fluxes and pressure gradients of oxygen and carbon dioxide can be derived as shown below:

$$
\begin{aligned}
\boldsymbol{q}_{O_{2}} & =\boldsymbol{\kappa}^{\mathrm{O}_{2} / O_{2}} \nabla \rho_{O_{2}}+\boldsymbol{\kappa}^{\mathrm{O}_{2} / C O_{2}} \nabla \rho_{C O_{2}} \\
\boldsymbol{q}_{C O_{2}} & =\boldsymbol{\kappa}^{C O_{2} / O_{2}} \nabla \rho_{O_{2}}+\boldsymbol{\kappa}^{C O_{2} / C O_{2}} \nabla \rho_{C O_{2}}
\end{aligned}
$$

where, $\boldsymbol{\kappa}^{A / B}$ denotes the micro-scale diffusivity relating the mass flux of species A with respect to the pressure gradient of species $\mathrm{B}$ and is computed as follows:

$$
\begin{aligned}
\kappa^{O_{2} / O_{2}} & =-P_{O_{2}} \frac{1}{\phi \mu_{g}} \mathbf{k}-\frac{D}{\phi} \varphi^{A} \frac{P_{C O_{2}}}{P} \\
\kappa^{O_{2} / C O_{2}} & =\frac{M_{O_{2}}}{M_{C O_{2}}}\left(-P_{O_{2}} \frac{1}{\phi \mu_{g}} \mathbf{k}+\frac{D}{\phi} \varphi^{A} \frac{P_{O_{2}}}{P}\right) \\
\kappa^{C O_{2} / O_{2}} & =\frac{M_{C O_{2}}}{M_{O_{2}}}\left(-P_{C O_{2}} \frac{1}{\phi \mu_{g}} \mathbf{k}+\frac{D}{\phi} \varphi^{A} \frac{P_{C O_{2}}}{P}\right) \\
\kappa^{C O_{2} / C O_{2}} & =-P_{C O_{2}} \frac{1}{\phi \mu_{g}} \mathbf{k}-\frac{D}{\phi} \varphi^{A} \frac{P_{O_{2}}}{P}
\end{aligned}
$$

The computational approach used for solve for the micro-scale field and flux distribution using the Taylor and homogenization approaches are listed below:

1. Micro-scale model - Taylor approach: The oxygen and carbon dioxide density field distributions in the micro-scale are directly obtained from the macro-scale solution using the Taylor boundary conditions (Eq. 10). The fluxes at all points within the microstructure are subsequently calculated using Eq. 25. Note that the Taylor solution may not satisfy the micro-scale equilibrium (Eq. 18).

2. Micro-scale model - Homogenization approach: Boundary conditions (Eq. 13) are specified in each unit cell. The two coupled non-linear partial differential equations (Eq. 18), one equation each for $\mathrm{O}_{2}$ and $\mathrm{CO}_{2}$ ) are solved using finite element analysis. To solve these nonlinear equations, Galerkin finite element method is adopted and the weak form is solved in an incremental iterative manner using the Newton-Raphson method. 


\section{Macro-scale model}

The macroscopic simulation is performed using a diffusion equation that is defined on a uniformly meshed domain $(\Omega)$ with boundaries defined as $\Gamma$. Degrading interfaces are explicitly modeled at the micro-scale, while only homogenized transport equations are modeled at the macro-scale as given below:

$$
\begin{array}{r}
\frac{\partial \bar{\rho}_{O_{2}}}{\partial t}+\nabla \cdot \overline{\boldsymbol{q}}_{O_{2}}=\bar{Q}_{O_{2}} \\
\frac{\partial \bar{\rho}_{C O_{2}}}{\partial t}+\nabla \cdot \overline{\boldsymbol{q}}_{C O_{2}}=-\bar{Q}_{C O_{2}} \\
\bar{\rho}(\Gamma, t>0)=\hat{\rho}, \quad \bar{\rho}(\Omega, t=0)=\bar{\rho}_{0}
\end{array}
$$

where, $\bar{\rho}$ is the macroscopic (homogenized) partial density and $\bar{Q}_{i}$ is the homogenized mass consumption rate of species $i$ per unit bulk volume defined as (where $f$ is the volume fraction of the burnt carbon fiber at the micro-scale):

$$
\bar{Q}_{i}=\rho_{c} \frac{M_{i}}{M_{c}} \frac{\partial f}{\partial t}
$$

To solve the non-linear transient macroscopic equations (Eq. 27), Galerkin finite element method and backward Euler time integration are adopted and the weak form is solved in an incremental iterative manner using the Newton-Raphson method. The $(\lambda+1)^{t h}$ Newton-Raphson step at time $(t+1)$ involves solution of the system $\boldsymbol{K}\left\{\delta \overline{\boldsymbol{\rho}}^{\lambda+1, t+1}\right\}=\boldsymbol{f}$, where the unknown vector in the above system is the increment in the partial density $\left(\delta \overline{\boldsymbol{\rho}}^{\lambda+1, t+1}\right)$ of oxygen and carbon dioxide. In our numerical approach, the reference density $\rho_{\text {ref }}$ and consumption rate $\bar{Q}$ (of each species $i$ ) for the next time step are evaluated at the end of each time step of the simulation. The reference density $\rho_{\text {ref }}$ is obtained using the macro-micro balance of mass condition $\left(\bar{\rho}=\frac{1}{V} \int_{V} \rho d V\right)$ and Eq. 1. This definition is consistent with the condition that stored mass at macro-scale is same as the average micro-scale stored mass [15]. To further understand the micro-scale quantities that are needed to create the overall system of equations, the Jacobian matrix and force vector for a finite element $e$ with shape functions $N_{i}$ occupying a volume $\Omega^{e}$ are expanded below:

$$
\begin{aligned}
& K_{i j}^{t+1, \lambda}=\left[\begin{array}{cc}
\frac{1}{\Delta t} \int_{\Omega^{e}} N_{i} N_{j} d V-\int_{\Omega^{e}} \frac{d N_{i}}{d x_{p}} \bar{\kappa}_{p q}^{O_{2} / O_{2}} \frac{d N_{j}}{d x_{q}} d V & -\int_{\Omega^{e}} \frac{d N_{i}}{d x_{p}} \bar{\kappa}_{p q}^{O_{2} / C O_{2}} \frac{d N_{j}}{d x_{q}} d V \\
-\int_{\Omega^{e}} \frac{d N_{i}}{d x_{p}} \bar{\kappa}_{p q}^{C O_{2} / O_{2}} \frac{d N_{j}}{d x_{q}} d V & \frac{1}{\Delta t} \int_{\Omega^{e}} N_{i} N_{j} d V-\int_{\Omega^{e}} \frac{d N_{i}}{d x_{p}} \bar{\kappa}_{p q}^{C O_{2} / C O_{2}} \frac{d N_{j}}{d x_{q}} d V
\end{array}\right] \\
& f_{i}^{t+1, \lambda}=\left[\begin{array}{c}
-\int_{\Omega^{e}} N_{i} \frac{\bar{\rho}_{O_{2}-\bar{\rho}_{O_{2}}^{t}}}{\Delta t} d V+\int_{\Omega^{e}} \frac{d N_{i}}{d x_{p}} \bar{q}_{O_{2} p} d V+\int_{\Omega^{e}} N_{i} \bar{Q}_{O_{2}}^{t} d V \\
-\int_{\Omega^{e}} N_{i} \frac{\bar{\rho}_{C O_{2}}-\bar{\rho}_{C O_{2}}^{t}}{\Delta t} d V+\int_{\Omega^{e}} \frac{d N_{i}}{d x_{p}} \bar{q}_{C O_{2} p} d V-\int_{\Omega^{e}} N_{i} \bar{Q}_{C O_{2}}^{t} d V
\end{array}\right]
\end{aligned}
$$

From the above equations, it is seen that homogenized diffusivities $\overline{\boldsymbol{\kappa}}^{A / B}$ relating the mass flux of species A with respect to the pressure gradient of species B needs to be defined at each integration point in the macro-scale. The evaluation of the homogenized diffusivity for Taylor and Homogenization approaches are listed below: In the Taylor model, the diffusivity is directly obtained by comparing Eq. 10, Eq. 25 and Eq. 16 as:

$$
\overline{\boldsymbol{\kappa}}^{A / B}=\frac{1}{V} \int_{\Omega} \boldsymbol{\kappa}^{A / B} d V
$$

In the homogenization approach, the homogenized diffusivity is obtained using perturbation analysis [23]. To aid in speeding up the solution process for the multi-scale problem, the algorithm was parallelized using MPI. The macro-scale domain was decomposed and elements in each domain distributed to different processors. The underlying micro-scale problems were solved in serial in each processor. The simulator was developed using object oriented programming and was dynamically linked to the parallel toolbox PetSc [24] for parallel assembly and solution of linear systems. For 
solution of linear systems, a GMRES solver along with block Jacobi and ILU preconditioning from the PetSc toolbox was employed.

VI. Tracking of the interface using level set method and adaptive meshing

Level set approach provides a natural way of tracking the degrading interface at the micro-scale during FE computations. We employ the stabilized Galerkin formulation on unstructured adaptive grids for solving the level set equation. The numerical implementation of the level set method is same as that detailed in Ref. [25]. The mass loss rate of oxygen has been calibrated as an Arrhenius equation using experimental results in [26]. To convert the measured mass loss rate to the velocity of oxidation at nodal points in the micro-scale oxidizing interface, we assume that the intact carbon fiber of initial radius $r_{0}$ oxidizes to a smaller radius $r$ after a short duration $\Delta t$. The velocity of recession at any point can then be found by scaling the mass loss rate with the initial as well as current radius $r_{o}$ and $r$ as below:

$$
\boldsymbol{v}=\frac{d r}{d t} \boldsymbol{n}=\frac{r_{0}^{2}}{2 r} k_{0} e^{\left(\frac{-E_{a}}{R T}\right)}\left(\frac{P_{O_{2}}}{P_{O_{2}}^{*}}\right)^{k_{1}} \boldsymbol{n}
$$

The experimentally calibrated parameters, $E_{a}, k_{0}, k_{1}$ from Ref. [4] are listed in Table 2. We approximate the current radius $r$ using the area of current carbon fiber $A$ as $r=\sqrt{\frac{A}{\pi}}$. Note that an inert coating (pyrolitic carbon) is generally applied on the carbon fiber to provide oxidation resistance. For simplicity, the ensuing simulations assume that the coating is not present and the carbon fiber is fully exposed to oxygen. Nevertheless, the effect of coatings can be easily included in this model by using a different recession velocity (Eq. 30) for the coating material.

Table 2 List of material constant for the carbon fiber oxidation example.

\begin{tabular}{|c|c|c|c|}
\hline Material constant & Matrix & Void & C-fiber \\
\hline Volumetric porosity & 0.1 & 1 & 0 \\
\hline$\frac{\text { Permeability }}{\text { Viscosity }}\left(\frac{\mathrm{m}^{2}}{\mathrm{kPa}}\right)$ & $10^{-7}$ & 0 & 0 \\
\hline Areal porosity & 0.00018 & 1 & 0 \\
\hline$M_{\mathrm{O}_{2}}(\mathrm{~g} / \mathrm{mol})$ & \multicolumn{3}{|c|}{32} \\
\hline$M_{\mathrm{CO}_{2}}(\mathrm{~g} / \mathrm{mol})$ & \multicolumn{3}{|c|}{14} \\
\hline$M_{\mathrm{C}}(\mathrm{g} / \mathrm{mol})$ & \multicolumn{3}{|c|}{6452.35} \\
\hline$k_{0}\left(\mathrm{~s}^{-1}\right)$ & 118300 \\
\hline$E_{a}(\mathrm{~J} / \mathrm{mol})$ & 3740 \\
\hline$\rho_{c}\left(\mathrm{~kg} / \mathrm{m}^{3}\right)$ & 3.5 \\
\hline$r_{0}(\mu \mathrm{m})$ & \multicolumn{3}{|c}{} \\
\hline
\end{tabular}

VII. Computational approach and Numerical Results

The overall solution scheme is shown in Table. 3. Homogenization and Taylor model differ in the way the micro-scale problem is solved, with the homogenization approach accounting for micro-scale equilibrium.

In this section, we focus on the problem of oxidation of an assembly of carbon fibers within a single carbon tow. Scanning electron micrographs of cross section of carbon tows reported in Refs. [27, 28] (Fig. 3(b)) reveal arrangement of carbon fibers in a hexagonal lattice within a tow. Micrographs obtained at high magnifications show the oxidation of individual carbon fibers (Fig. $3(c)$ ) as well as the preferential oxidation of carbon fibers adjoining the porous matrix due to high oxygen availability. As the outer fibers oxidize, oxygen diffuses inward along the voids formed and react with the interior fibers. The oxidized carbon fiber front (Fig. 3(b)) evolves inward into the 
(1) Initialize macro-scale model and assign a microstructure (and FE meshes) to every integration point.

(2) Apply time increment $\Delta t$ to the macro-scale problem.

(3) Iteration step:

(3.1) Assemble the macroscopic stiffness matrix. (3.2). Solve the macroscopic system and compute density and the

(3.3) Loop over all integration points set method.

(a) (Only done for the first NR iteration) Update the carbon fiber using level

(b) Taylor model: Transfer densities to every point in the micro-scale mesh using Eq. 10.

Homogenization model: Transfer boundary conditions to micro-scale problem. Assemble and solve the micro-scale problem.

(c) Calculate the volume averaged macro-flux (Eq. 16), the source term (Eq. 28) and the macro-diffusivity.

(3.4) Assemble the macroscopic residual vector.

(4) Check convergence, if not converged go to step 3, otherwise go to step 2.

carbon fiber tow, until all the fibers are oxidized. In our numerical model, a section of carbon fiber tow (with about 600 carbon fibers) is taken to be our macroscopic model. The size of our macroscopic simulation cell (shown in Fig. 4(a)) is indicated using dotted lines on the micrographs. In the macroscopic model, the evolution of homogenized carbon density is tracked as oxygen diffuses from the left end to the right end. All the boundaries in the model are insulated (as shown in Fig. 3(a)) except one side that is exposed to the external environment of pure oxygen with imposed pressures of $P_{\mathrm{O}_{2}}=0.1 \mathrm{~atm}$ and $P_{\mathrm{CO}_{2}}=0 \mathrm{~atm}$ for $t>0$. The system is modelled at a constant temperature of $950^{\circ} \mathrm{C}$. An initial condition (at $t=0$ ) of $P_{\mathrm{O}_{2}}=0.1 \mathrm{~atm}$ and $P_{\mathrm{CO}_{2}}=0 \mathrm{~atm}$ is imposed at all points in the specimen. The micro-scale physics of carbon fiber oxidation is introduced by modeling a single carbon fiber within a hexagonal unit cell as shown in Fig. 4(b). Since the carbon fibers do not mediate the diffusion of the gas species, the carbon fiber is not modeled in the microscale. The unit cell is assigned to each integration point in the macro-grid.

Simulation of carbon tow oxidation in the configuration depicted in Fig. 3(a) was carried out using both Taylor and Homogenization approaches. In general, simulations reveal diffusion controlled kinetics at the beginning of oxidation. As the carbon fibers oxidize, a void is created in place of the oxidized carbon fiber. This greatly increases subsequent diffusion of oxygen towards the interior of the carbon tow. Due to increased oxygen availability, a transition from diffusion controlled kinetics to reaction controlled kinetics is seen. This is exemplified by a linear rate law, ie. oxidized carbon volume fraction increasing linearly with respect to time. A mixed rate law of the following form describes the overall oxidation process:

$$
\frac{\sqrt{x}}{k_{r}}+\frac{x}{k_{l}}=t
$$

where, $k_{r}$ and $k_{l}$ are the rate constants. The reaction rate constants predicted by the Taylor and Homogenization approaches are quite different as seen in Table. 4. The Taylor model provides a overconstrained response compared to FE homogenization approach due to strict imposition of oxygen and carbon dioxide densities within the unit cell. In fact, the Taylor model provides an upper bound response for system, an effect well studied in literature (eg. [13]). The Taylor model, as expected, predicts faster oxygen transport within the tow compared to the FE homogenization approach. FE homogenization approach solves the micro-scale equilibrium equations and the micro- 

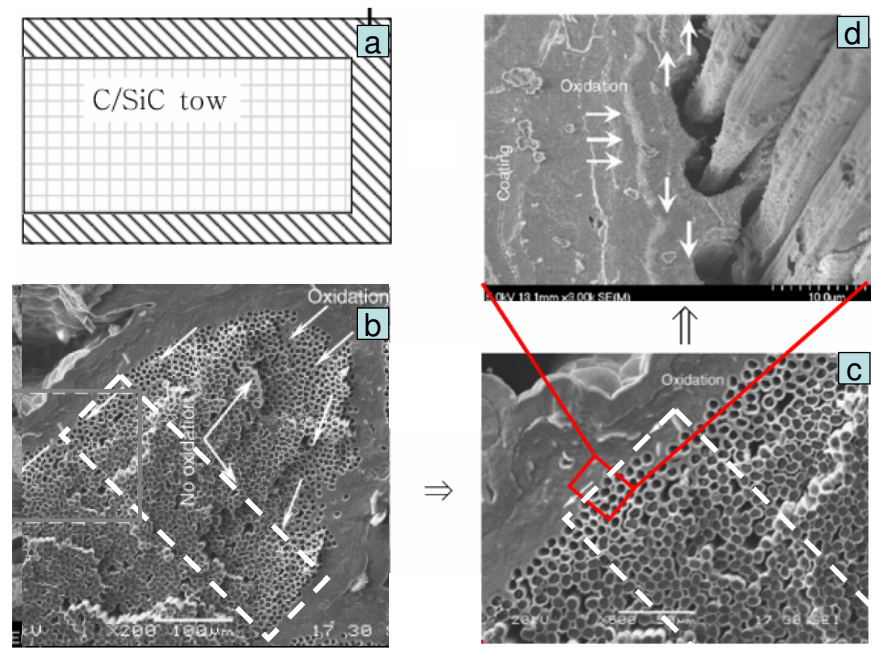

Fig. 3 The micrographs represent experimental results of $C / S i C$ composite oxidation in a controlled environment reported in [27] and [28]. Figure (d) shows the simulation cell: all the boundaries in the model are insulated except one side that is exposed to the external oxidizing environment. Size of the simulation cell at the macro-scale is indicated using dotted lines in $(b)$. The simulation cell contains about 600 carbon fibers.

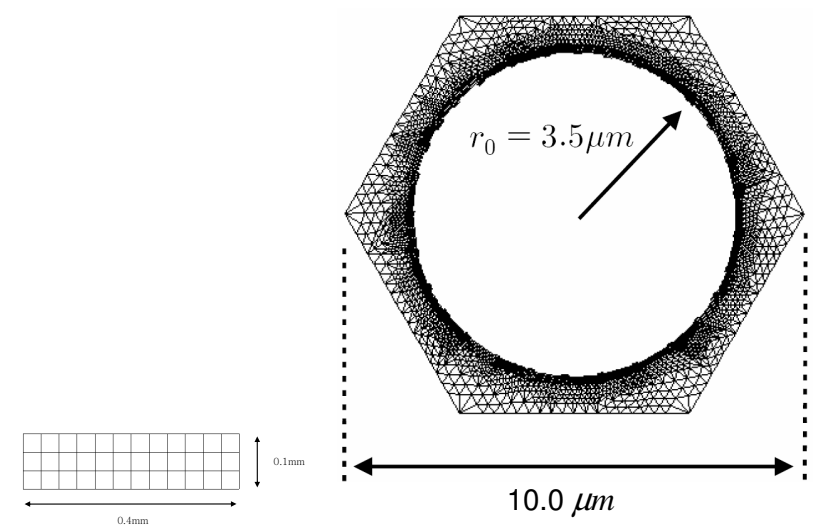

(a)

(b)

Fig. 4 (a) 2D Macro-scale finite element grid (b) Micro-scale finite element grid

scale solution is more physically reasonable. However, the choice of selection of one model versus another is based on the relative importance of accuracy versus computational speed. In the Taylor model, there is no need for finite element computations at the micro-scale and the convergence of the overall non-linear multiscale scheme is faster. In the case considered here, Taylor model was solved four times faster than the homogenization model.

Table 4 Square root and linear rate constants obtained by curve fitting the simulation results

\begin{tabular}{|c|c|c|c|}
\hline & $k_{r} \times 10^{4}\left(1 / s^{2}\right)$ & $k_{l} \times 10^{2}(1 / s)$ & Transient time(sec) \\
\hline Taylor & 13.7 & 3.8 & 13.1 \\
\hline Homogenization & 8.4 & 2.5 & 13.1 \\
\hline
\end{tabular}

In order to illustrate changes in the carbon fiber configuration in the micro-scale, the Taylor model solution at selected 6 integration points are shown in Fig. 5 at a time of 25.1 seconds. Although the macro-problem studied corresponds to a one dimensional diffusion, the micro-scale 
oxidation results shown in Fig. 5 do not follow this trend. Indeed, the one dimensional nature of the macroscopic density gradient is considered in the micro-scale simulation through the scale linking equation (Eq. 1). However, the characteristic length scale over which the macroscopic field variables vary is much larger than the size of the microscopic volume considered. Consequently, the contribution of the macroscopic gradient term to the micro-scale oxygen and carbon dioxide density (ie. term $\nabla \rho \cdot \boldsymbol{x}$ in Eq. 1) is not significant compared to the macroscopic oxygen density term (term $\rho_{\text {ref }}$ in Eq. 1). In other words, the macroscopic oxygen density field translates to a nearly constant oxygen density at the level of the RVE. This kind of uniform oxidation of carbon fibers is indeed seen from experimental micrographs as shown in Fig. 3(d).

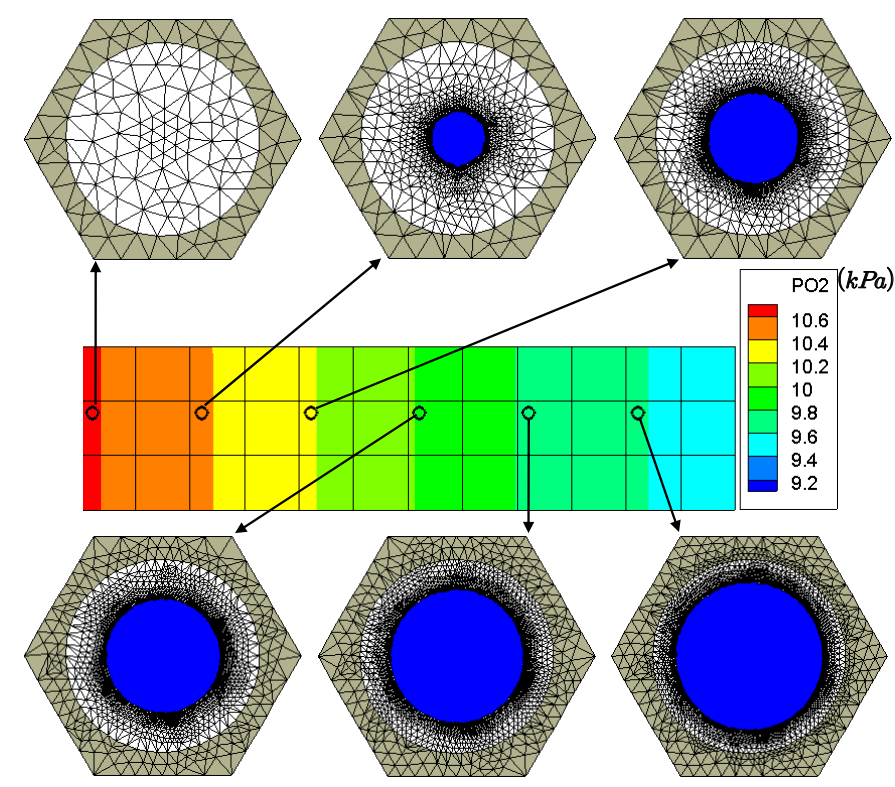

Fig. 5 Carbon fiber configurations in micro at 25.1 seconds at $950^{\circ} \mathrm{C}$; tan, white and blue colored area indicate matrix, void and carbon fiber respectively.

A comparison of the simulation results from the Taylor and homogenization approaches at various locations in the macroscopic mesh at a simulation time of 25.1 seconds is shown in Fig. 6. In this figure, the tan, white and blue colored areas indicate matrix, void and carbon fiber respectively. Both Taylor and homogenization models indicate complete oxidation of the carbon fibers located closest to the exposed macro-scale surface (left edge) at 25.1 seconds. The oxygen partial pressure solution at the micro-scale shows the contrast between the two approaches. The boundary conditions in the Taylor model lead to enforcement of high oxygen densities within the matrix compared to the physically accurate solution from homogenization that satisfies micro-scale equilibrium. In the steady state micro-scale solution, the oxygen density in the void (that is left behind after oxidation of the carbon fiber) is expected to be uniform due to much faster diffusion in the void compared to the porous matrix. This is clearly seen from the homogenization approach, while in the Taylor model, a variation of oxygen density in the void is enforced. Further, in all cases, the Taylor model provides an upper bound for the oxygen density solution (and faster oxidation) at both macro- and micro-scales. The Taylor model predicts partial oxidation while homogenization approach predicts an almost intact carbon fiber at an integration point located on the right end.

\section{Conclusion}

In this paper, a non-linear coupled macro-micro finite element model is presented for addressing carbon fiber oxidation problems. Oxidation involves evolution of carbon fiber surfaces coupled with flux jump boundary conditions across interfaces that have not been addressed using homogenization approaches. Homogenization of complex micro-scale behavior including moving interfaces and flux jumps has been performed in this work. A nested finite element solution scheme is implemented into a multiscale analysis framework. The results from these two multi-scaling approaches 


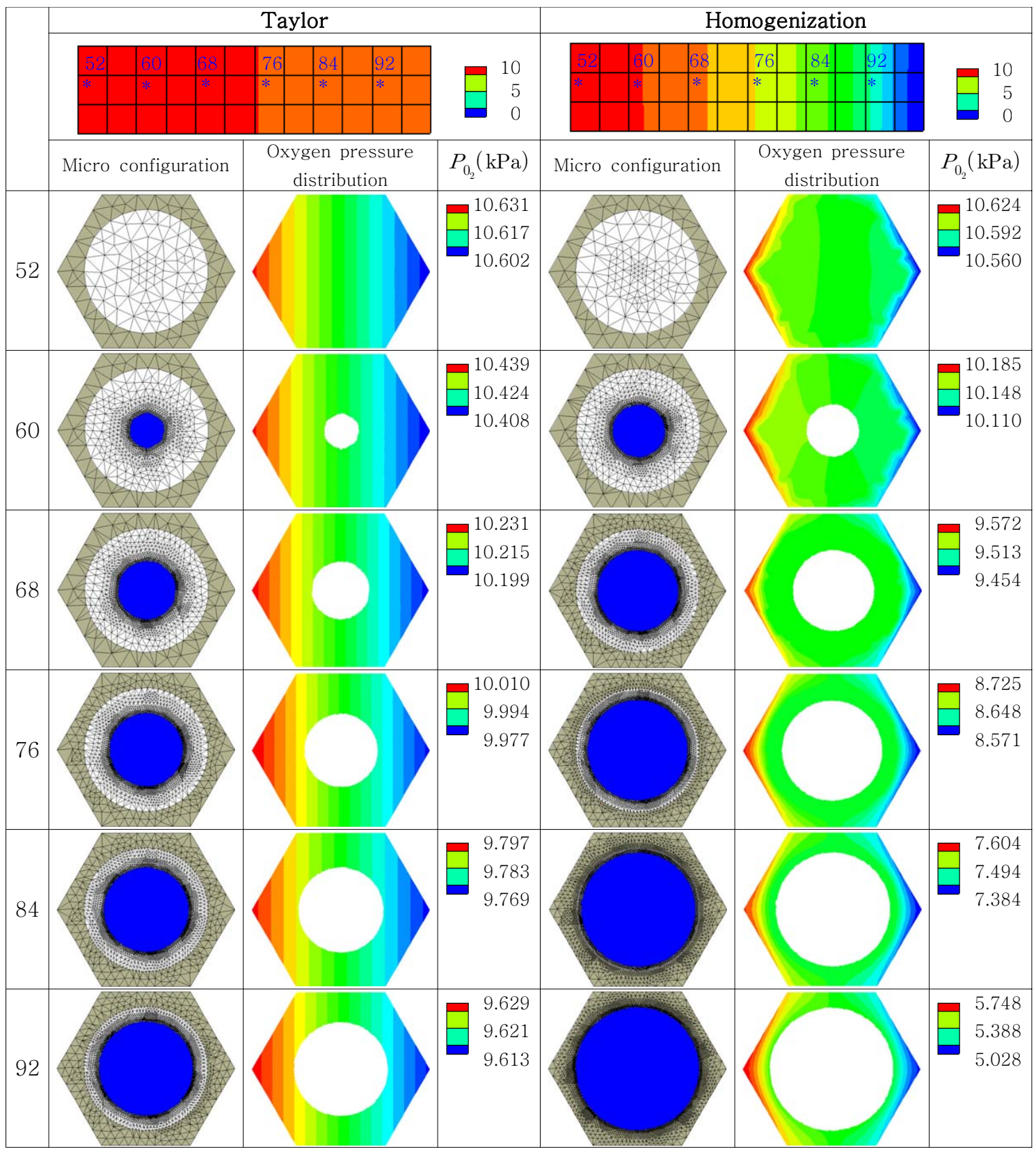

Fig. 6 Comparison of carbon fiber configuration and micro-scale oxygen densities for Taylor and homogenization approaches at a simulation time of 25.1 seconds.

were compared for a simple one dimensional oxidation of a carbon fiber tow. In both models, the influence of microstructural evolution (e.g. carbon fiber oxidation) on the competition between macroscopic oxygen and carbon dioxide transport in the macro-scale can be introduced and treated effectively. Taylor model is computationally efficient but provides an upper bound response and predicts faster oxygen transport within the tow compared to the FE homogenization approach. The proposed method, when combined with thermo-mechanical field effects, constitutes a powerful tool, for modeling high temperature oxidation of composites. The homogenization approach enhances the understanding and modeling of micro-scale interfacial phenomena and in the future, would allow modeling of interesting mechanisms such as interaction of porosity evolution with carbon fiber oxidation ('stress-oxidation coupling') that cannot be explained without incorporating microstructural details. 
This work was sponsored by NASA Constellation University Institutes Project under grant NCC3-989 with Claudia Meyer as the project manager.

\section{References}

[1] Glass DE, Ceramic Matrix Composite (CMC) Thermal Protection Systems (TPS) and Hot Structures for Hypersonic Vehicles, 15th AIAA Space Planes and Hypersonic Systems and Technologies Conference. AIAA-paper 2008-2682, 28 April-1 May 2008, Dayton, Ohio.

[2] Eckel AJ, Cawley JD, Parthasarathy TA, Oxidation Kinetics of a Continuous Carbon Phase in a Nonreactive Matrix. Journal of the American Ceramic Society 1995;78(4):972-980

[3] Halbig MC. The oxidation kinetics of continuous carbon fibers in a cracked ceramic matrix composite. NASA/TM-2001-210520. 2001.

[4] Sullivan RM, A model for the oxidation of carbon silicon carbide composite structures. Carbon $2005 ; 43(2): 275-285$

[5] Hashin Z. Analysis of composite materials. Journal of Applied Mechanics 1983;50:481-505.

[6] Rosen BW, Hashin Z. Effective thermal expansion coefficients and specific heats of composite materials. International Journal of Engineering Science 1970;8:157-173.

[7] Noor AK, Shah RS. Effective thermoelastic and thermal properties of unidirectional fiber-reinforced composites and their sensitivity coefficients. Composite Structures 1993;26:7-23.

[8] Auriault JL. Effective macroscopic description of heat conduction in periodic composites. International Journal of Heat and Mass Transfer 1983; 26(6):861-869.

[9] Guedes JM, Kikuchi N. Preprocessing and postprocessing for materials based on the homogenization method with adaptive finite element methods. Computer Methods in Applied Mechanics and Engineering 1990;83:143-198.

[10] Jiang M, Jasiuk I, Ostoja-Starzewski M. Apparent thermal conductivity of periodic two-dimensional composites. Computational Materials Science 2002; 25:329-338.

[11] Smit RJM, Brekelmans WAM, Meijer HEH. Prediction of the mechanical behaviour of nonlinear heterogeneous systems by multi-level finite element modeling. Computer Methods in Applied Mechanics and Engineering 1998;155:181-192.

[12] Miehe C, Schroder J, Schotte J. Computational homogenization analysis in finite plasticity. Simulation of texture development in polycrystalline materials. Computer Methods in Applied Mechanics and Engineering 1999;171:387-418.

[13] Sundararaghavan V, Zabaras N. Design of microstructure-sensitive properties in elasto-viscoplastic polycrystals using multi-scale homogenization. International Journal of Plasticity 2006;22:1799-1824

[14] Belytschko T, Loehnert S, Song JH, Multiscale aggregating discontinuities: a method for circumventing loss of material stability. International Journal for Numerical Methods in Engineering 2008;73:869Ŭ-894.

[15] Lee S, Sundararaghavan V. Multiscale modeling of moving interface problems with flux jumps: Application to solidification. Computational Mechanics, 2009;44(3):297-307.

[16] Hill, R., On constitutive macro-variables for heterogeneous solids at finite strain. Proc. Roy. Soc. London, 1972;326A:131-147.

[17] J. T. Ward, J. B. Hamblen, Influence of Diffusion of Oxygen on the Rate of Combustion of Solid Carbon, Ind. Eng. Chem., 1927;19(9):1025Ü-1027.

[18] Ozdemir I, Brekelmans WAM, Geers MGD. Computational homogenization for heat conduction in heterogeneous solids. Int. J. Numer. Meth. Engng. 2008;73:185-204

[19] Rao VS, Hughes TJR, Garikipati K. On modelling thermal oxidation of Silicon II: numerical aspects. Int. J. Numer. Meth. Engng. 2000;47: 359-377.

[20] LeVeque R. Numerical Methods for Conservation ÿaws. Birkhauser: Basel, 1992.

[21] Bird RB, Stewart WE, Lightfoot EN. Transport phenomena. New York, NY: Wiley; 1960.

[22] Bacos MP, Dorvaux JM, Lavigne O, Talandier J. C/C Composite oxidation model III. Physical basis, limitations and applications. Carbon 2000;38(1):105-117.

[23] Miehe C. Numerical computation of algorithmic (consistent) tangent moduli in large-strain computational inelasticity, Computer Meth. Applied Mech. and Engg. 1996; 134(3-4):223-240.

[24] Balay, S, Buschelman, K, Eijkhout, V, Gropp, WD, Kaushik, D, Knepley, MG, McInnes, LC, Smith, BF, Zhang, H, 2004. PETSc Users Manual ANL-95/11 - Revision 2.1.5, Argonne National Laboratory.

[25] Tan L, Zabaras N. A level set simulation of dendritic solidification of multi-component alloys. Journal of Computational Physics 2007;221(1):9-40.

[26] Halbig MC. Carbon oxidation studies and the evaluation of oxidation inhibited C/SiC composites. 28th annual conference on composites, materials and structures Cocoa Beach (Florida, USA): United States Advanced Ceramics Association, 2004.

[27] Mei H, Cheng L, Zhang L, Damage mechanisms of C/SiC composites subjected to constant load and thermal cycling in oxidizing atmosphere. Scripta Materialia 2006;54:163-168 
[28] Mei H, Cheng L, Zhang L, Thermal Cycling damage mechanisms of C/SiC composites in displacement constraint and oxidizing atmosphere. Journal of the American Ceramic Society 2006;89(7):2330-2334.

[29] Verrilli M, Calomino A. Temperature dependence on the strength and stress rupture behavior of a carbon fiber-reinforced silicon carbide matrix (C/SiC) composite. Ceram. Eng. Sci. Proc. 2003;24(4):443449.

[30] Ostoja-Starzewski M. Towards stochastic continuum thermodynamics. Journal of Non-equilibrium Thermodynamics 2002;27:335-348. 